\title{
Role of Hematological Indices in COVID-19 Patient's Prognosis
}

\author{
Chakrapani Mittal ${ }^{1}$, Aviral Gupta ${ }^{2}$, Puneet Rijhwani ${ }^{3}$, Anchin Kalia ${ }^{4}$, Shrikant Choudhary ${ }^{5}$, Mukesh Sarna ${ }^{6}$, Aakriti Vij $^{7}$
}

\begin{abstract}
Aim and background: In this study, the role of hematological indices in coronavirus disease 2019 (COVID-19) patients' prognosis who have visited our hospital has been evaluated.

Materials and methods: Clinical profile, disease severity, disease outcome, blood parameters [hemoglobin, white blood cell counts, neutrophil counts, lymphocyte counts, neutrophil:Iymphocyte ratio (NLR), lymphocyte:monocyte ratio, platelet counts, eosinophil count, and platelet:lymphocyte ratio (PLR)] were evaluated in patients with confirmed COVID-19 infection.

Results: High values of leukocytes, neutrophils, and low value of platelets, eosinophil counts indicated poor prognosis in COVID-19 patients. High NLR, PLR, and low lymphocyte:monocyte ratio also indicated a bad prognosis.

Conclusion: Monocyte count, lymphocyte count, eosinophil count, NLR, lymphocyte monocyte ratio, and PLR values can be used in the prognosis of COVID-19 patients.

Keywords: Coronavirus disease 2019, Hematological indices, Neutrophil:lymphocyte ratio, Platelet:lymphocyte ratio.

Journal of Mahatma Gandhi University of Medical Sciences \& Technology (2021): 10.5005/jp-journals-10057-0155
\end{abstract}

\section{INTRODUCTION}

On December 29, 2019, pneumonia cases were detected in a hospital in Wuhan, China.' The Chinese Center for Disease Control and Prevention studied throat cultures from these patients later confirmed that these cases were caused by a new type of betacoronavirus. ${ }^{2}$ The mode of transmission of the disease is person-toperson transmission via droplet infection and indirect contact. ${ }^{3}$ The information about the disease is ever-evolving and new methods of prognostication and lines of management are constantly being evaluated.

Coronaviruses are pathogenic and cause respiratory, enteric, neurological, and hepatic diseases. ${ }^{4}$ The elderly and specific patient groups, such as those with underlying medical conditions are at risk of severe disease. ${ }^{5}$

The time of onset of acute respiratory distress syndrome after hospital admission is short, which makes early diagnosis and prognostication critical due to the high mortality rates in the coronavirus disease 2019 (COVID-19). ${ }^{6}$

Complete blood counts (CBC) assays are readily available, inexpensive, easy to perform, and highly automated. The assay includes values such as total white blood cell count, absolute neutrophil count, lymphocyte and platelet counts, mean platelet volume, and certain ratios of these values, such as neutrophil:lymphocyte ratio (NLR), lymphocyte:monocyte ratio, and platelet:lymphocyte ratio (PLR), which can be used as markers of physiological stress. Neutrophils are an important component of the immune system and are the most characteristic cell type among the white blood cells. They take part in the inflammatory processes and are regulated by mast cells epithelial cells and macrophages. There is a clearly evident role of lymphocytes in both inflammation and infections. Platelets also have a role in the regulation of various inflammatory processes and are an acute phase reactant. While these blood cells may be used as inflammatory markers alone, their ratios to one another maybe even more useful indicators of

\footnotetext{
${ }^{1-7}$ Department of General Medicine, Mahatma Gandhi Medical College and Hospital, Jaipur, Rajasthan, India

Corresponding Author: Aviral Gupta, Department of General Medicine, Mahatma Gandhi Medical College and Hospital, Jaipur, Rajasthan, India, Phone: +91 9871199853, e-mail: aviral.aiims@gmail. com

How to cite this article: Mittal C, Gupta A, Rijhwani P, et al. Role of Hematological Indices in COVID-19 Patient's Prognosis. J Mahatma Gandhi Univ Med Sci Tech 2021;6(2):39-41.

Source of support: Nil

Conflict of interest: None
}

early inflammation. ${ }^{7-9}$ Circulating white blood cells respond to stress (such as infections) by increasing neutrophils and reducing lymphocytes; thus, the ratio of these two parameters (NLR) can be used as an inflammatory marker. ${ }^{10}$

\section{Materials and Methods}

\section{Study Design and Participants}

This single-center retrospective observational study was conducted at Mahatma Gandhi Medical College and Hospital, Jaipur, Rajasthan. One hundred and eighty-seven patients diagnosed with COVID19 were included in the study. The study was approved by the Institutional Ethics Committee, Mahatma Gandhi Hospital, Jaipur. The need for informed consent from patients was waived due to the time constraints during the COVID-19 emergency.

\section{Data Collection}

We collected data of 187 patients from their medical records. These data include age, sex, clinical symptoms, levels of illness severity (asymptomatic, mild, moderate, and severe), outcome, and hematological indices (hemoglobin, white blood cell count, 
neutrophil count, lymphocyte count, platelet count, eosinophil count, NLR, lymphocyte:monocyte ratio, and PLR). All the data were reviewed and analyzed by senior physicians.

\section{Statistical Analysis}

Normally distributed continuous variables are expressed as means \pm standard deviations, and non-normally distributed continuous variables as medians and interquartile ranges. Meanwhile, categorical variables are described as frequencies and percentages. The independent $t$-test or Mann-Whitney $U$ test was conducted to compare continuous variables between the groups of patients. Meanwhile, the $\chi^{2}$-test or Fisher's exact test was used to analyze the associations between categorical variables. A $p<0.05$ was considered as statistically significant, and all statistical analyses were performed using Epi-info software.

\section{Results (Tables 1 to 4 )}

Table 1: Clinical profile

\begin{tabular}{|c|c|c|}
\hline \multicolumn{2}{|c|}{ Variable } & Frequency \\
\hline \multicolumn{2}{|c|}{ Age in years (median, IQR) } & $47(33-62)$ \\
\hline \multirow[t]{2}{*}{ Sex } & Male $n(\%)$ & $130(69.52)$ \\
\hline & Female $n(\%)$ & $57(30.48)$ \\
\hline \multicolumn{2}{|c|}{ Fever $n(\%)$} & $107(57.21)$ \\
\hline \multicolumn{2}{|c|}{ Cough $n(\%)$} & $90(48.13)$ \\
\hline \multicolumn{2}{|c|}{ Headache $n(\%)$} & $34(18.18)$ \\
\hline \multicolumn{2}{|c|}{ Malaise $n(\%)$} & $66(35.29)$ \\
\hline \multicolumn{2}{|c|}{ Shortness of breath $n$ (\%) } & 63 (33.69) \\
\hline
\end{tabular}

Table 2: Severity of disease

\begin{tabular}{ll}
\hline Variable & Frequency \\
\hline Asymptomatic $n(\%)$ & $44(23.53)$ \\
Mild $n(\%)$ & $93(49.73)$ \\
Moderate $n(\%)$ & $20(10.70)$ \\
Severe $n(\%)$ & $30(16.04)$ \\
\hline
\end{tabular}

Table 3: Outcome

\begin{tabular}{lc}
\hline Variable & Frequency \\
\hline Death $n(\%)$ & $22(11.76)$ \\
ICU stay (median, IQR) & $9.50(8.0-12.0)$ \\
\hline
\end{tabular}

\section{Discussion}

In the results of this study, low thrombocyte, leukocyte, and neutrophil counts were revealed in COVID-19 positive patients. These results are consistent with previous research. Guan et al. noted thrombocytopenia and leukopenia in their study. ${ }^{11}$

Assiri et al. also noted the presence of thrombocytopenia in their study ${ }^{12}$ and leukopenia was also noted in another study conducted by Xu et al. ${ }^{13}$ In general, leukocytosis $(>10,000)$ was seen in bacterial pneumonias, with a leukocyte count of $>50,000$ in some cases; and leukocyte count was lower than 10,000 in viral pneumonias. Xu et al. found significant thrombocytopenia in pneumonia patients, and the decrease in platelet counts was directly proportional to the patients' clinical status. ${ }^{14}$ In a study by Fan et al., COVID-19 positive patients had mild thrombocytopenia and leukopenia at first admission. ${ }^{15}$ Viral pneumonia affects the immune system leading to a decrease in platelet count, leukocyte count, and neutrophil counts.

The NLR in peripheral blood has been used to distinguish between bacterial and viral infections. ${ }^{16,17}$ Our study population had a median NLR of 12.24 in the fatal group, as compared to 2.30 for the survival group ( $p$ value 0.001 ). In a retrospective study of patients hospitalized with a fever of an unknown origin, it was shown that NLR is higher in those with bacterial infections as compared to those with fever due to a viral etiology. ${ }^{18}$

In the study by Zhang et al., NLR taken within 24 hours of admission was used as an early prognostic marker for AIV-H7N9 patients, with the NLR in the fatal group being significantly higher than the survival group. ${ }^{19}$ Yang et al. found that with a cut-off of 3.3 for NLR, there was a specificity of 0.636 , and a sensitivity of 0.88 for NLR in determining the prognosis for seriously ill COVID-19 patients with an AUC of $0.743 .{ }^{20}$

The eosinophil count in the mortality group was significantly lower than in the patients who survived COVID-19 in our study ( $p$ value 0.01 ). Tanni et al. ${ }^{21}$ noted the complete absence of eosinophils ( 0 eosinophils) in $88 \%$ of COVID-19 patients in the first 2 days of admission, and $86 \%$ of the deceased patients who initially presented with eosinopenia remained eosinopenic.

\section{Conclusion}

In this study, low values of lymphocytes, monocyte, and eosinophil count were found with a CBC test which is easily available in the hospital were found to be valuable in terms of the severity of COVID-19. In addition, high values of NLR, LMR, and PLR were also indicative of a bad prognosis of COVID-19.

Table 4: Hematological indices

\begin{tabular}{|c|c|c|c|c|}
\hline Variable & Total $(n=187)$ & Death $(n=22)$ & Survival $(n=165)$ & $p$ value \\
\hline $\mathrm{Hb}$ (median) (IQR) & $12.10(6.12-16.24)$ & $11.92(9.81-13.64)$ & $12.72(6.10-16.24)$ & 0.36 \\
\hline WBC counts (median) (IQR) & $6,600(5,540-8,700)$ & $8,700(5,780-1,360)$ & $6,400(5,190-7,830)$ & 0.14 \\
\hline Neutrophil count (median) (IQR) & $4,325(2,320-6,000)$ & $7,700(4,920-10,900)$ & $4,005(2,900-5,850)$ & 0.07 \\
\hline Lymphocyte count (cells/ $\mu \mathrm{L}$ ) (median) (IQR) & $1,548(1,040-6,620)$ & $610(480.0-980.0)$ & $1,600(1,200-6,620)$ & 0.03 \\
\hline NLR (median) (IQR) & $4.87(1.57-5.43)$ & $12.24(6.10-16.50)$ & $2.30(1.44-4.36)$ & 0.001 \\
\hline Monocyte count (median) (IQR) & $395(280-580)$ & $280(180-600)$ & $400(290-600)$ & 0.001 \\
\hline Lymphocyte monocyte ratio (median) (IQR) & $4.11(2.57-6.06)$ & $2.05(0.84-3.78)$ & $4.35(2.75-6.46)$ & 0.001 \\
\hline PLR (median) (IQR) & $125.55(89.79-185.16)$ & $215.68(136.92-300)$ & $119.73(87.89-171.78)$ & 0.001 \\
\hline Platelets (median) (IQR) & $1.89(1.50-2.52)$ & $1.34(1.11-1.78)$ & $1.93(1.55-2.57)$ & 0.06 \\
\hline
\end{tabular}




\section{References}

1. Li Q, Guan X, Wu P, et al. Early transmission dynamics in Wuhan, China, of novel coronavirus-infected pneumonia. N Engl J Med 2020;382(13):1199-1207. DOI: 10.1056/NEJMoa2001316.

2. Lu H, Stratton CW, Tang YW. Outbreak of pneumonia of unknown etiology in Wuhan, China: the mystery and the miracle. J Med Virol 2020;92(4):401-402.

3. Prevention CfDCa, First travel-related Case of 2019 Novel Coronavirus Detected in United States. U.S. Department of Health \& Human Services 2020 [Available from: http://www.cdc.gov/media/ releases/2020/p0121-novel-coronavirus-travel-case.html.

4. WHO, Statement on the second meeting of the International Health Regulations (2005) Emergency Committee regarding the outbreak of novel coronavirus (2019-nCoV) 2020 [Available from: https://www.who. int/news/item/30-01-2020-statement-on-the-second-meeting-ofthe-international-health-regulations-(2005)-emergency-committeeregarding-the-outbreak-of-novel-coronavirus-(2019-ncov).

5. Bennett JDR, Blaser MJ. Principles and practice of infectious diseases. 8th ed., PA, USA: Elsevier/Saunders; 2014.

6. Lu R, Yu X, Wang W, et al. Characterization of human coronavirus etiology in Chinese adults with acute upper respiratory tract infection by real-time RT-PCR assays. PLoS One 2012;7(6):e38638. DOI: 10.1371/ journal.pone.0038638.

7. Bekdas M, Goksugur SB, Sarac EG, et al. Neutrophil/lymphocyte and $\mathrm{C}$-reactive protein/mean platelet volume ratios in differentiating between viral and bacterial pneumonias and diagnosing early complications in children. Saudi Med J 2014;35(5):442-447.

8. Ilhan M, Ilhan G, Gok AF, et al. Evaluation of neutrophil-lymphocyte ratio, platelet-lymphocyte ratio and red blood cell distribution width-platelet ratio as early predictor of acute pancreatitis in pregnancy. J Matern Fetal Neonatal Med 2016;29(9):1476-1480. DOI: 10.3109/14767058.2015.1051026.

9. Yazar FM, Bakacak M, Emre A, et al. Predictive role of neutrophil-tolymphocyte and platelet-to-lymphocyte ratios for diagnosis of acute appendicitis during pregnancy. Kaohsiung J Med Sci 2015;31(11):591596. DOI: 10.1016/j.kjms.2015.10.005.

10. Xiang N, Havers $F$, Chen T, et al. Use of national pneumonia surveillance to describe influenza A(H7N9) virus epidemiology, China, 2004-2013. Emerg Infect Dis 2013;19(11):1784-1790. DOI: 10.3201/ eid1911.130865.
11. Guan WJ, Ni ZY, Hu Y, et al. Clinical characteristics of coronavirus disease 2019 in China. N Engl J Med 2020;382(18):1708-1720. DOI: 10.1056/NEJMoa2002032.

12. Assiri A, Al-Tawfiq JA, Al-Rabeeah AA, et al. Epidemiological, demographic, and clinical characteristics of 47 cases of Middle East respiratory syndrome coronavirus disease from Saudi Arabia: a descriptive study. Lancet Infect Dis 2013;13(9):752-761. DOI: 10.1016/ S1473-3099(13)70204-4.

13. $X u X W$, Wu XX, Jiang $X G$, et al. Clinical findings in a group of patients infected with the 2019 novel coronavirus (SARS-Cov-2) outside of Wuhan, China: retrospective case series. BMJ 2020;368:m606. DOI: 10.1136/bmj.m606.

14. $X u Y$, Zhang $Y$, Jiang $F$, et al. Comparison of relevant indicators of coagulation and fibrinolysis in patients with varying severity of community-acquired pneumonia. Zhonghua Yi Xue Za Zhi 2015;95(24):1925-1929.

15. Fan $\mathrm{BE}$, Chong VCL, Chan SSW, et al. Hematologic parameters in patients with COVID-19 infection. Am J Hematol 2020;95(6):E131E134. DOI: 10.1002/ajh.25774.

16. Loonen AJM, de Jager CPC, Tosserams J, et al. Biomarkers and molecular analysis to improve bloodstream infection diagnostics in an emergency care unit. PLoS One 2014;9(1):e87315. DOI: 10.1371/ journal.pone.0087315.

17. Yoon NB, Son C, Um SJ. Role of the neutrophil-lymphocyte count ratio in the differential diagnosis between pulmonary tuberculosis and bacterial community-acquired pneumonia. Ann Lab Med 2013;33(2):105-110. DOI: 10.3343/alm.2013.33.2.105.

18. Naess A, Mo R, Nilssen SS, et al. Infections in patients hospitalized for fever as related to duration and other predictors at admittance. Infection 2014;42(3):485-492. DOI: 10.1007/s15010-013-0573-1.

19. Zhang $Y$, Zou $P$, Gao H, et al. Neutrophil-lymphocyte ratio as an early new marker in AIV-H7N9-infected patients: a retrospective study. Ther Clin Risk Manag 2019;15:911-919. DOI: 10.2147/TCRM. S206930.

20. Yang AP, Liu JP, Tao WQ, et al. The diagnostic and predictive role of NLR, d-NLR and PLR in COVID-19 patients. Int Immunopharmacol 2020;84:106504. DOI: 10.1016/j.intimp.2020.106504.

21. Tanni F, Akker E, Zaman MM, et al. Eosinopenia and COVID-19. J Am Osteopath Assoc 2020. DOI: 10.7556/jaoa.2020.0910nline ahead of print. 